\title{
Atopic Dermatitis: Preventing and Managing The Itch That Rashes, and A Case For The Multi-Theory Model (MTM) for Health Behavior Change for Educational Interventions
}

Kayla Penny, M3 ${ }^{1}$, Manoj Sharma, MBBS, PhD, MCHES® ${ }^{2}$, Amy E. Flischel, MD³, Robert T. Brodell, $\mathrm{MD}^{3}$, Vinayak K. Nahar MD, PhD, $\mathrm{MS}^{3,4}$

${ }^{1}$ LSU Health Shreveport School of Medicine, Shreveport, LA

2 Department of Environmental \& Occupational Health, School of Public Health, University of Nevada, Las Vegas, NV

${ }^{3}$ Department of Dermatology, School of Medicine, University of Mississippi Medical Center, Jackson, MS

${ }^{4}$ Department of Preventive Medicine, School of Medicine/John D. Bower School of Population Health, University of Mississippi Medical Center, Jackson, MS

\section{ABSTRACT}

Atopic dermatitis (AD) is a multifactorial disease affected by a host of genetic, environmental, socioeconomic and demographic influences, that induce the atopic immune response in predisposed individuals. Despite treatment with topical corticosteroids to reduce skin inflammation, emollients to improve the skin barrier, and avoidance of inflammatory triggers, many patients report progressive symptoms. Management strategies are developed as modifiable environmental influences are identified. Managing atopic dermatitis requires adaptive changes in health behavior involving the patient and often the caregiver. Multi-theory models (MTM) have not yet been used in AD interventions but may prove beneficial as they use behavior concepts to predict both initiation and sustenance in education health interventions. A comprehensive approach fosters such changes by using psychological and educational strategies as adjuncts to conventional therapy. This article reviews the challenges in managing $A D$ and the potential impact of behavioral theories. This is designed to strengthen the argument for using an MTM model in future studies of AD.

\section{Atopic dermatitis}

underlying etiology of atopic dermatitis is still not completely understood,

Atopic dermatitis (AD), or eczema, is the most common chronic noncontagious inflammatory skin condition in the world (1). It presents with chronic relapsing pruritic patches and plaques in a distinct distribution and is associated with other atopic conditions (asthma and allergic rhinitis). While the

it is thought to be multifactorial, involving genetic, psychosocial, and environmental factors, a defective skin barrier, and altered immunologic responses, whereby, predisposed individuals develop cutaneous hypersensitivity to environmental antigens. 


\section{Signs and symptoms of AD in children}

The diagnosis of $A D$ is made primarily on clinical grounds. Severe pruritus is a universal symptom and erythematous, scaly or lichenified, excoriated papules and plaques are commonly seen (2). These findings are distributed in age-specific patterns. Babies present with a papulosquamous morphology on facial and extensor surfaces, which gravitates to flexor creases as patients age. The signs and symptoms are associated with the itchscratch cycle. Pruritus leads to scratching that damages the integrity of the skin barrier producing inflammation that causes more pruritus. The absence of filaggrin, a key moisture-protective element in the stratum corneum, enables antigens associated with external pathogens to penetrate the skin exacerbating the allergic response.

\section{Consequences of $A D$ in children}

The distressing, chronic, waxing and waning course of $A D$ severely impacts quality of life. Chamlin et al. (2004) studied the impact of AD on children and their families. Nearly 180 quality-of-life outcomes were identified and grouped by domains as related to physical health, emotional health, physical functioning, and social functioning (3). The effects on the child and the family were assessed in each domain. The physical health and physical functioning domains had the most deleterious effects on the child. Pruritus and scratching can lead to pain, bleeding and sleep disturbance. Other studies have demonstrated that sleep disturbances resulting from atopic dermatitis also carry a psychosocial burden reflected by drowsiness, inability to focus, irritability, and growth restriction (4). Impacts on physical functioning include clothing restrictions, interference with indoor and outdoor activities such as bathing and swimming, and embarrassment (5).

While the effects of physical health and functioning more directly impact the child suffering from $A D$, emotional influences often produce social effects that impact both children and families. For example, children with atopic dermatitis who display behavioral problems and irritability may interact less with other children (social impairment) (5). In addition, the emotional and social impacts on families may be worsened by the economic burden carried by families of children with $A D$. These annual expenditures can be categorized as: 1) direct costs including expenditures on prescription drugs, OTC products, inpatient and outpatient medical visits totaling $\$ 1.009$ billion; and 2) indirect costs including the loss of earnings due to time away from work for caregivers totaling $\$ 619$ million. In addition, willingness to pay for relief of symptoms or intangible costs equaled $\$ 2.6$ billion (5) (6).

\section{Prevalence of $A D$ in children}

AD affects individuals of all ages and backgrounds. Of the 16.5 million people in the U.S affected by $A D, 9.6$ million are children with $80 \%$ having onset of disease before 6 years of age (2). AD is known to predominantly impact industrialized nations. In the U.S, the prevalence of AD has risen from $8 \%$ to $12 \%$ since 1997 . Furthermore, studies show that there is a $50 \%$ lower risk for developing $A D$ in children who were born in countries outside of the US, but the risk increases after living in the US for a decade (2). There is greater prevalence in households with increased incomes and higher education (6). Additionally, smaller family sizes and residing in urban and metropolitan areas appear to increase the prevalence of $A D(6)$. 
Public health significance of managing and preventing complications of $A D$ in children

AD produces a substantial public health burden, partly driven by its impact on the quality of life but also resulting from the high healthcare costs and utilization associated with disease management. The relentless symptoms decrease productivity and the desire for relief produces direct, indirect, and tangible costs for both patients and payers. Amongst skin diseases, AD demonstrates the fourth highest quality of life-related costs (5). Compared to hypertension and diabetes, $A D$ in the United States has \$371-\$489 greater out-of-pocket cost per person per year (2). Unfortunately, these costs represent an even greater burden for the underprivileged. In fact, $17.6 \%$ of patients delay care and $13.1 \%$ of patients do not even seek medical care for $A D$ due to concerns about health care expenses (2). In addition, $15.7 \%$ of patients who seek care are unable to pay the cost of prescriptions (2). Identifying approaches to prevent and manage mild $A D$ is a focus of public health measures.

\section{Determinants of $A D$ in children including the role of parents}

While studies regarding the public health burden imposed by $A D$ define the geographical and socioeconomic influences on this condition, research must also focus on causative factors and determinants of disease severity. $A D$ is certainly multifactorial involving a host of genetic and environmental influences. The genetic influence is supported by twin studies. (7). Environmental antigens associated with $A D$ include allergens associated with house-dust mites and food, as well as non-allergenrelated factors, such as irritating clothing material, Staphylococcus aureus infections, microorganism exposure at a young age, and heat (8). Environmental allergens impacted by socioeconomic and demographic influences, shift a genetically predisposed individual towards the development of an AD immune response. Several factors are associated with more severe disease: neglected housing, parental education level, general health of the mother, parental emotional health, lower household income, single parent home, and being the firstborn child (9). As modifiable determinants such as these are determined, specifically those linked to the role of parenting, new management strategies can be developed.

\section{Self-management of $A D$ in children: challenges}

The current standard of care for $A D$ involves the use of topical corticosteroids to reduce skin inflammation, emollients to promote skin hydration and protect the skin barrier, and efforts to avoid inflammatory triggers. Despite these efforts, many patients report untreated symptoms half of the time during flares (10). Additionally, fewer than $25 \%$ of both caregivers and patients report confidence in their ability to control AD flares (10). It is, therefore, important to consider the factors that lead to non-adherence to treatments. A cross-sectional survey of mothers and children with $A D$ in Tokyo determined both disease severity and a stable, healthy doctorpatient relationship predicted adherence to treatment (11). The trusted physician may mitigate misunderstandings related to treatment regimens. Another study assessed the relationship between the concern for topical steroid side effects and adherence in AD patients. Unwarranted concern of side effects led $39 \%$ of patients and caregivers to use topical corticosteroids for shorter durations and less frequently than recommended. Up to $66 \%$ of patients reported using topical steroids only as a last resort (10). In fact, the biggest challenge 
faced in self-management of AD results from a gap between physicians and patient's/caregiver's knowledge about treatment. At least two-thirds of caregivers and patients believe that the ability to confidently control $A D$ is the most important determinant of quality of life (10). This individual belief or sense of confidence in the ability to control a situation is termed "selfefficacy." When parents believe they have the ability to successfully control the $A D$ of their child, this is known as parental selfefficacy (12). As research surrounding childhood chronic diseases, such as asthma and cystic fibrosis, has advanced the concept of parenteral self-efficacy (PSE). PSE predicts the performance of management tasks in parents of children with chronic disease and has been found to predict morbidity (12). Thus, the perceptions of patients and caregivers about the management of their $A D$ are associated with their self-efficacy and likelihood of treatment adherence. For this reason, it would be expected that $A D$ management could be improved by testing educational methods that utilize self-efficacy and task performance measurements as indicators of efficacy (12, 13).

Educational interventions for parental involvement in self-management of $A D$ in children

For over three decades, educational interventions have been developed to effectively manage AD. These have focused on knowledge acquiring processes, including teaching and learning utilizing reinforcement. The goal of these interventions is to give patients a better understanding of their disease processes and management principles by providing treatment instructions and prevention strategies related to the effective management of AD (14). They also aim to provide parents with self-efficacy, which has the potential to predict their performance of disease-specific management tasks (12).

A meta-analysis of educational intervention trials in AD patients published in 2020 was one of the first to demonstrate the relationship between education and managing $A D$ on a large scale. Using a comprehensive search of databases, researchers identified studies that met the following characteristics and conditions: participants with ages from one month to 18 years, presence of a health education group and a control group, and outcomes that were measured using SCORAD (severity scoring of atopic dermatitis index) and quality of life indices such as Infants' Dermatology Quality of Life Index (IDQOL) $(15,16)$. These studies demonstrated that health education interventions played a significant role on IDQOL score, increasing at 3 and 6 months and SCORAD scores significantly decreased at 6 and 12 months when compared to a control group (15). However, these trials were carried out in different countries and failed to include characteristics such as patient demographics and the education strategy utilized. Additional efficacy studies followed by multi-centric effectiveness educational trials with large sample sizes are needed (15).

\section{Limitations of educational interventions}

Although positive outcomes have been associated with educational programs, there are limitations with current educational approaches and the tools used to measure their success. Specifically, parental educational interventions for children with $A D$ often do not incorporate the education of the child. This is a missed opportunity since AD outcome measures are almost always childcentered (severity of disease and patient quality of life). On the other hand, Mitchell et 
al. noted that child-centered outcomes may be less sensitive indicators of success when education is parent-focused (12). Thus, parent-specific outcome variables such as self-efficacy and outcome expectations are more reliable and valid indicators when evaluating interventions with parental focus. In a systematic review, Ersser et al. support a similar notion by emphasizing that parents are the primary emphasis of the educational modalities, while children are the primary emphasis of psychological approaches (14).

This systematic review by Ersser et al. analyzed the two main adjunctive approaches to pharmaceutical therapy for $A D$ : psychological and educational interventions. Both educational and psychological interventions, when used as separate entities, have major drawbacks and indicate the need for more comprehensive intervention strategies. Specifically, educational interventions that lack a psychologic interventional component are flawed and fail to provide the tested approaches that allow parents to develop long-lasting, meaningful behavioral changes in their children with AD. The missing elements that are required to induce behavior change include factors such as participant motivation with set goals, the provision of confidence and autonomy in decisionmaking, and the development of problemsolving skills that promote the achievement of goal-directed action plans (14).

To improve educational interventions, methods must be developed that draw a connection between intention and behavior change. Self-efficacy theory-based educational interventions provide patients with the knowledge, behavior-specific skills, and confidence needed to cope with their condition successfully (14). For example, an elementary education program focused on changing physical activity through the social cognitive theory should have concrete goals. After the intervention, $80 \%$ of the participants should demonstrate improvement in: 1) physical activity expectations; 2) physical activity self-efficacy scoring; and 3) physical activity self-control score (17). The objectives listed here would not be measured in an interventional program that is purely educational. Studies on AD should not rely so heavily on self-management, and a comprehensive intervention creating a longlasting behavioral impact is a more important goal.

\section{Application of Behavioral Theories in the Management of AD}

Chronic diseases require life-long selfmanagement techniques that are adaptable, yet durable. Educational programs seek to understand why patients develop healthpromoting behaviors, leading to hopefully more effective interventions (18). Several theories have evolved to promote healthy behaviors. The 2010 Annual Review of Public Health lists the most popular approaches: The Health Belief Model (HBM), , Social Cognitive Theory (SCT), Transtheoretical Model (TTM) and Social-Ecological Model (SEM) (18). The HBM focuses on the perception of risks and benefits to disease prevention and development. On the other hand, the TTM and SCT are related to disease prevention and management. While the TTM outlines stages of change for successful health behaviors, the SCT promotes change based on an individual and family experiences. Finally, the Social Ecological Model emphasizes the environments that are most suitable for changes to take place.

The Berlin parental educational program uses behavioral theory and utilizes the underlying principles of the Social Learning Theory (SLT, the older version of SCT) to identify behavioral elements required 
for the patient to adapt and gain the knowledge necessary for change (19). In addition, the SLT stresses self-efficacy as an essential component in developing cognitive changes that facilitate desired health behaviors (19). With the goal of improving parent's self-management skills, these principles are integrated with educational objectives regarding the medical, nutritional, and psychological basis for effective management of AD (19). The six-week program consists of weekly 2-hour sessions run by pediatricians, psychologists, and dieticians that incorporate educational objectives (medical, psychological, and nutritional information) with skill training and open group discussions (19). Medical topics emphasize the causes and treatment of $A D$, appropriate over-the-counter skin care, and the avoidance of environmental triggers. The psychological aspect of the program highlights the management of stress and the itch-scratch cycle while providing coping mechanisms for both the child and family. Finally, nutritional topics assess the associated risks and benefits of dietary programs, avoidance of allergies tied to $A D$, and provide nutritional recommendations. SLT is tied to educational goals utilizing the following techniques: 1) skills training that includes goal setting and putting desired behaviors into small steps; and 2) modeling; 3) positive reinforcement; and, 4) monitoring of behaviors and environmental triggers (19). $A$ randomized control trial utilizing the Berlin educational program was instituted in 7 German hospitals. Parents of children with $A D$ were stratified by age of the child into 3 different experimental groups. Results demonstrated that, compared to control groups, all three intervention groups had significant improvements in symptom severity (20).

A case for the multi-theory model (MTM) of health behavior change for educational
Interventions for parental involvement in self-management of $A D$ in children

Evidence shows that incorporating theoretical fundamentals into interventions improves overall efficacy (19). Furthermore, the application of interventions based on multiple theories can result in additive impacts on efficacy (18). The Berlin parental educational program which focused on $A D$ and several other public health interventions, confirmed that utilizing behavioral theories in conjunction with educational interventions can instill long-standing principles of disease self-management. Researchers, however, may refrain from incorporating multiple theories for several reasons. For example, measuring and analyzing constructs of certain health behavior theories is challenging (18). Teaching large amounts of information may overshadow the role of theory in an intervention (18). Finally, considering the number of theoretical frameworks in the literature today, it may be impossible to find one that suits an educational interventional program perfectly.

The ideal theory will predict health behavior change that is both immediate and long-term and produced by sufficient evidence with adequate predictive power. The constructs must be modifiable and retain value for individuals, groups, and the community across various cultures. $(21,17)$. Regrettably, the perfect theory has yet to be developed. For this reason, the SLT theory, which expands upon multiple disciplines and self-efficacy, is frequently chosen for health interventions designed to affect behavior changes in conditions such as AD. Using the SLT theory alone, however, disregards important elements which could be incorporated with behavioral theories. For example, The TTM model is most specific to behavior change compared to all other health promotion and health education approaches 
(21). Using the TTM, however, is associated with some disadvantages. For example, because TTM uses arbitrary stages, it can be quite ambiguous, making it difficult to apply several of its constructs (21). Furthermore, although it is inherent to behavior change, the TTM has an underlying context of psychotherapy and fails to address health education measures (21). HBM, while focused on health education, addresses change at the level of the individual and has limited predictability. The shortcomings of individual-level behavioral theories have led to multi-theory models of health education intervention in chronic diseases such as AD.

Health behavior change is dependent on initiating modification and maintaining the behavioral change. Sharma emphasizes the constructs initiating change are not the same as the constructs needed to maintain behavior change (21). Existing theories which explain behavior change do not make this distinction and may account for lower predictive power (21). This led to the multitheory model (MTM) for health behavior change pulling together several constructs that are derived from successful behavioral theories, while permitting each to operate independently from one another. The model derived by Sharma is composed of six constructs, three focused on initiating behavior change and three directed at maintaining behavior change. The constructs which address initiating behavior change are: a dialogue about the pros and cons of behavior change, self-efficacy and its impact on confidence, and components of the physical environment that make a behavioral change feasible (17; See Figure 1 for adaptation of this model to self-management behaviors for atopic dermatitis). The constructs which maintain change are emotional transformation, reflection and reflective action around change, and a social environment with a support system for behavioral change (21; See Figure 2 for adaptation of this model to self-management behaviors for atopic dermatitis).

Interventions for parents of children with $A D$ must initiate and maintain behavior changes. Although the MTM for health behavior has not been studied specifically for interventions in $A D$, it has been utilized for other health interventions that identified predictive factors associated with initiation and sustenance of these interventions.

A cross-sectional study using MTM in the US demonstrated the model's ability to predict both initiation and maintenance of anxietyreducing behaviors in students (22). The analysis was performed with MTM constructs used as the independent variables and intention to initiate and maintain relaxation behaviors as dependent variables. There were significant associations between behavioral confidence with the initiation of relaxation behavior and emotional transformation with sustenance (22). A similar cross-sectional study sought to identify predictive factors for Ghanaian adolescents' intention receive HPV vaccination (23). Changes in physical environment was found to be a significant predictor of intention to accept the vaccine (initiation). Two significant predictors for completing the recommended doses of HPV vaccination (sustenance) were practice for change and emotional transformation. 


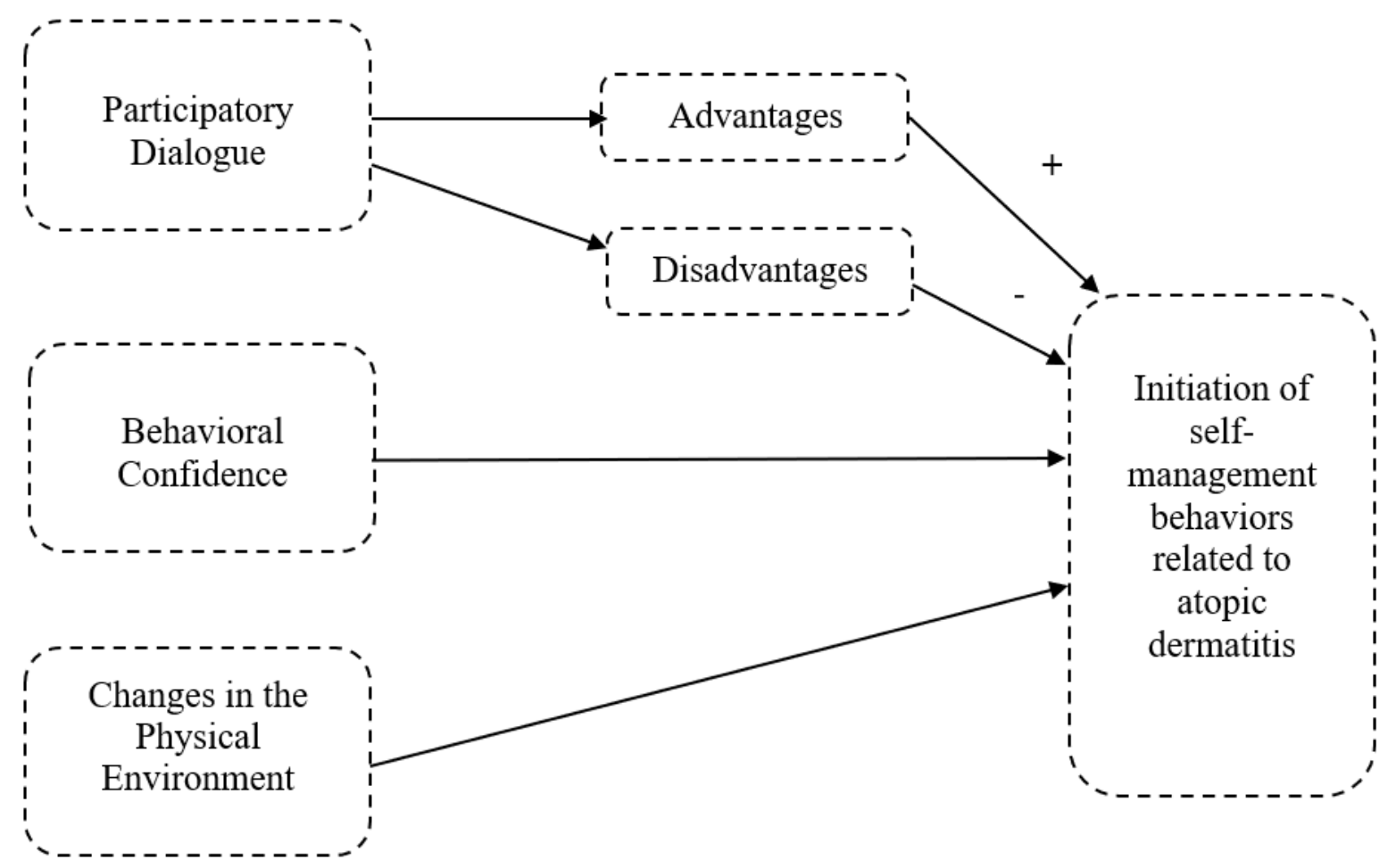

Figure 1. Diagrammatic depiction of the initiation model of MTM for changes in self-management behaviors related to atopic dermatitis

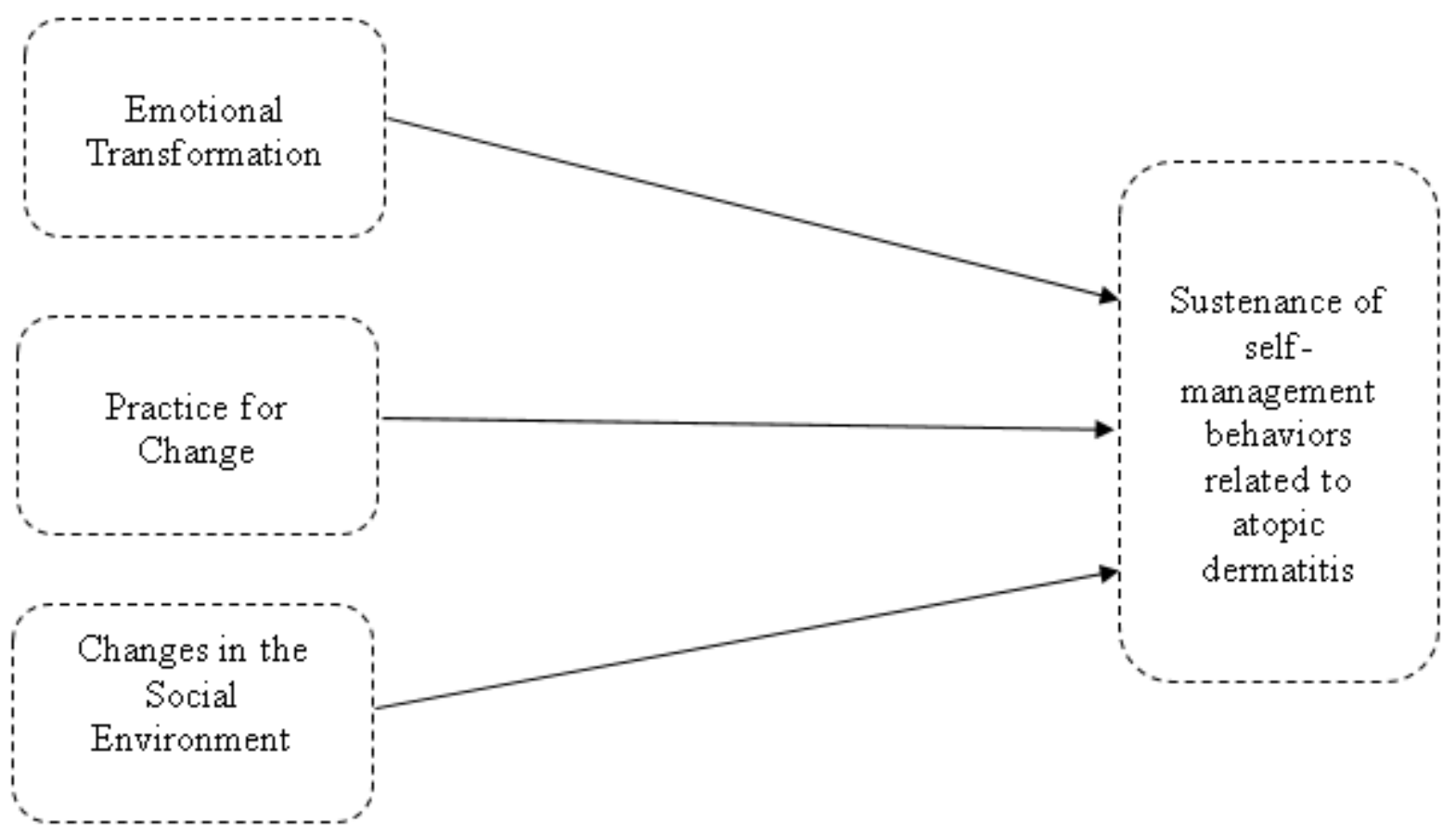

Figure 2. Diagrammatic depiction of the sustenance model of MTM for changes in self-management behaviors related to atopic dermatitis 
Interestingly, while physical environment and practice for change were found to be significant predictors in this study, another study utilizing the MTM model for anxiety in veterinary students found neither to be predictive. Differences in the external environment in each study may explain this phenomenon. The notion that emotional transformation remained significant for both studies with very different demographics indicates the possibility that this may be a more universal construct for predicting intention to change.

In addition to identification of constructs which may be positive predictors for initiation and sustenance, studies have also studied the extent to which certain constructs yield positive results. In a study that applied the multi-theory model (MTM) to predict water consumption in lieu of sugar-sweetened beverages (SSBs), researchers found that changes in the physical environment and behavioral confidence were associated with a $61.8 \%$ of the variance in the commencement of drinking water behavior instead of SSBs (24). Constructs of emotional transformation and practice for change were associated with a $58.3 \%$ variance in the sustenance of drinking water instead of SSBs (24). These findings provide additional support that changes in the physical environment and behavioral confidence as significant predictors for initiation and practice for change and emotional transformation as significant predictors for sustenance.

Utilization of the MTM for health behavior intentions goes beyond the identification of predictors of behavioral change. Several studies have assessed interventions using MTM models and yielded positive results. One study tested the efficacy of an MTMbased intervention on starting and maintaining physical activity among African American women and compared it to a traditional intervention based on mere knowledge acquisition (25). When compared to the knowledge-based intervention, results showed the MTM-based intervention was associated with significant increases in the minutes of physical activity, reductions in waist circumference, and modification of changes in the physical environment (25). While these results demonstrate the possibility for successful MTM based interventions, they also demonstrate the impact of modifying the environment based on demographics. Another study demonstrated the efficacy of MTM-based educational interventions on waterpipe smoking in male adolescent students (26). A statistically significant decrease in the frequency of waterpipe smoking in the intervention group was noted. The study also went further comparing constructs between study and control groups. Participatory dialogue, behavioral confidence, practice for change, and emotional transformation were all significantly different in the intervention arm compared to the control arm, demonstrating a clear connection between the success of the intervention and constructs of the MTM model for health behavior.

\section{Implications for clinical practice}

Since AD is largely a condition of childhood, the role of a parent/caregiver is critically important in disease management. If adequate attention is not given to the parentrelated exacerbating factors of $A D$ (such as emotional stress, inappropriate skin care technique, dietary restrictions, etc.), even patients with the most comprehensive management plans may fail to achieve significant improvement (27). The factors listed above, and knowledge and beliefs 
surrounding medications used in treatment regimens can be targeted in educational intervention programs. Furthermore, health educational interventions have shown efficacy in the management of several chronic diseases that impact adults directly, and as a result, have been used to target and improve the role of parents in managing ongoing childhood conditions such as AD. In addition to knowledge about the condition itself, other parental factors such as emotional health and self-efficacy are important determinants in $\operatorname{AD}(9,10)$. Furthermore, since $A D$ is an ongoing condition, a model that instills a long-standing behavioral change is needed. Similar to management principles for chronic conditions impacting adults, managing atopic eczema requires adaptive changes in health behavior promoting action of the patient and the caregiver (14). A comprehensive approach includes psychological and educational strategies as adjuncts to conventional therapy (14). For this reason, educational models that incorporate theories of behavior have the ability to make a more permanent and effective impact on various conditions requiring treatment adherence. Of course, no single behavioral theory targets both initiation and maintenance of health behavioral change (21). In addition, there are several behavioral constructs that underlie a caregiver's impact on $A D$ that can be addressed by various behavior theories therefore, MTM models for health education interventions may be of particular importance (18). Though not yet investigated in AD, MTM models for other health behavior interventions have shown evidence predictive of positive behavior change. In summary, MTM models may be important in the study of $A D$ because they not only bring together various behavioral constructs that affect the role of caregivers but also initiation and sustenance in educational health interventions.

\section{Recommendations for research}

Few studies exist focusing on behavioral theories that impact educational interventions for managing $A D$, and no studies have evaluated the use of an MTM model for AD interventions. To strengthen the argument for using this model in the study of $A D$, the impacts of behavioral theories in chronic diseases were analyzed in this article. Independent behavioral theories on educational interventions for AD suggest that utilizing multiple theories may lead to more successful interventions. In addition, the outcome variables used to assess the results should reflect both disease severity in the patient and behavior changes induced by the intervention. Thus, behavioral constructs that are significant predictors for initiation and sustenance should be identified and differentiated. This may be done with studies modeled after those that test the efficacy of the MTM model in predicting health behavioral change $(22,23)$. Finally, the results of these studies can be used to develop and assess interventions utilizing the MTM model to ensure that parents are able to manage their child's AD effectively. (25, 26)

Conflict of Interest Disclosures: Robert T. Brodell has participated in multi-center clinical trials with: Genentech, Janssen Pharmaceuticals, Corrona Psoriasis Registry, Novartis, and Glaxo-Smith-Kline. $\mathrm{He}$ is also associate editor of the Journal of the American Academy of Dermatology and editor-inchief of Practice Update: Dermatology. Kayla Penny, Manoj Sharma, Amy E. Flischel, and Vinayak K. Nahar have no conflicts of interest.

Funding: None

Corresponding Author:

Vinayak K. Nahar, MD, PhD, MS

Department of Dermatology

School of Medicine

University of Mississippi Medical Center

2500 North State Street - L216 
Jackson, MS 39216 USA

(601) 495-5876

naharvinayak@gmail.com

\section{References:}

1. Weidinger, S., Beck, L.A., Bieber, T. et al. Atopic dermatitis. Nat Rev Dis Primers 4, 1 (2018). https://doi.org/10.1038/s41572-018-0001-z

2. Eczema Facts. National Eczema Association. Retrieved June 1, 2020, from https://nationaleczema.org/research/eczemafacts/

3. Chamlin, S. L., Frieden, I. J., Williams, M. L., \& Chren, M. M. (2004). Effects of atopic dermatitis on young American children and their families. Pediatrics, 114(3), 607-611

4. Mian, M., Silfvast-Kaiser, A. S., Paek, S. Y., Kivelevitch, D., \& Menter, A. (2019). A Review of the Most Common Dermatologic Conditions and their Debilitating Psychosocial Impacts. International Archives of Internal Medicine, 3, 018

5. Drucker, A. M., Wang, A. R., Li, W. Q., Sevetson, E., Block, J. K., \& Qureshi, A. A. (2017). The burden of atopic dermatitis: summary of a report for the National Eczema Association. Journal of Investigative Dermatology, 137(1), 26-30

6. Silverberg, J. I. (2017). Public health burden and epidemiology of atopic dermatitis. Dermatologic Clinics, 35(3), 283-289.

7. Cookson, William O.C.M.; Moffatt, Miriam F. The genetics of atopic dermatitis, Current Opinion in Allergy and Clinical Immunology: October 2002 Volume 2 - Issue 5 - p 383-387

8. Williams, H., 2005. Atopic Dermatitis. New England Journal of Medicine, 352(22), pp.2314-2324.

9. Silverberg JI, Simpson EL. Associations of childhood eczema severity: a US population-based study. Dermatitis. 2014;25(3):107-114. doi:10.1097/DER.0000000000000034

10. 1Zuberbier, T., Orlow, S. J., Paller, A. S., Taïeb, A., Allen, R., Hernanz-Hermosa, J. M., ... \& Simon, J. C. (2006). Patient perspectives on the management of atopic dermatitis. Journal of Allergy and Clinical Immunology, 118(1), 226-232.

11. Ohya, Y., Williams, H., Steptoe, A., Saito, H., likura, Y., Anderson, R., \& Akasawa, A. (2001). Psychosocial factors and adherence to treatment advice in childhood atopic dermatitis. Journal of Investigative Dermatology, 117(4), 852-857.

12. Mitchell, A. E., \& Fraser, J. A. (2014). Management of atopic dermatitis in children: Evaluation of parents' self-efficacy, outcome expectations, and self-reported task performance using the Child Eczema Management Questionnaire. Neonatal, Paediatric and Child Health Nursing, 17(2), 16-22.
13. Ersser SJ, Latter S, Sibley A, Satherley PA, Welbourne S. Psychological and educational interventions for atopic eczema in children. Cochrane Database Syst Rev. 2007;(3):CD004054.

14. Ersser, S. J., Cowdell, F., Latter, S., Gardiner, E., Flohr, C., Thompson, A. R., ... \& Drury, A. (2014). Psychological and educational interventions for atopic eczema in children. Cochrane Database of Systematic Reviews, (1).

15. Li, Y., Han, T., Li, W., Li, Y., Guo, X., \& Zheng, L. (2020). Efficacy of health education on treatment of children with atopic dermatitis: a meta-analysis of randomized controlled trials. Archives of Dermatological Research, 1-11.

16. Charman $\mathrm{C}$, Williams $\mathrm{H}$. Outcome Measures of Disease Severity in Atopic Eczema. Arch Dermatol. 2000;136(6):763-769. doi:10.1001/archderm.136.6.763

17. Sharma M. Theoretical Foundations of Health Education and Health Promotion. Sudbury: Jones \& Bartlett Learning; 2017

18. Glanz, K., \& Bishop, D. B. (2010). The role of behavioral science theory in development and implementation of public health interventions. Annual Review of Public Health, 31, 399-418

19. Wenninger, K., Kehrt, R., von Rüden, U., Lehmann, C., Binder, C., Wahn, U., \& Staab, D. (2000). Structured parent education in the management of childhood atopic dermatitis: the Berlin model. Patient Education and Counseling, 40(3), 253-261

20. Staab D, Diepgen TL, Fartasch M, et al. Age related, structured educational programmes for the management of atopic dermatitis in children and adolescents: multicentre, randomised controlled trial. BMJ. 2006;332(7547):933-938. doi:10.1136/bmj.332.7547.933

21. Sharma, M. (2015). Multi-theory model (MTM) for health behavior change. Webmed Central Behaviour, 6(9), WMC004982

22. Nahar, V. K., Wells, J., Davis, R. E., Johnson, E. C., Johnson, J. W., \& Sharma, M. (2020). Factors Associated with Initiation and Sustenance of Stress Management Behaviors in Veterinary Students: Testing of Multi-theory Model (MTM). International Journal of Environmental Research and Public Health, 17(2), 1-10

23. Asare, M., Agyei-Baffour, P., Lanning, B. A., Barimah Owusu, A., Commeh, M. E., Boozer, K., ... \& Paskett, E. D. (2020). Multi-Theory Model and Predictors of Likelihood of Accepting the Series of HPV Vaccination: A Cross-Sectional Study among Ghanaian Adolescents. International Journal of Environmental Research and Public Health, 17(2), 571.

24. Sharma, M., Catalano, H. P., Nahar, V. K., Lingam, V., Johnson, P., \& Ford, M. A. (2017). Using MultiSeptember 2021 Volume 5 Issue 5 
Theory Model (MTM) of Health Behavior Change to Predict Plain Water Consumption Instead of Sugar

Sweetened Beverages. Journal of Research in Health Sciences, 17(1), e00370

25. Hayes, T., Sharma, M., Shahbazi, M., Sung, J. H., Bennett, R., \& Reese-Smith, J. (2019). The evaluation of a fourth-generation multi-theory model (MTM) based intervention to initiate and sustain physical activity in African American women. Health Promotion Perspectives

26. Bashirian, S., Barati, M., Sharma, M., Abasi, H., \& Karami, M. (2019).Water pipe smoking reduction in the male adolescent students: An educational intervention using multi-theory model. Journal of Research in Health Sciences, 19(1), e00438

27. LeBovidge, J. S., Elverson, W., Timmons, K. G., Hawryluk, E. B., Rea, C., Lee, M., \& Schneider, L. C. (2016). Multidisciplinary interventions in the management of atopic dermatitis. Journal of Allergy and Clinical Immunology, 138(2), 325-334. 